\title{
The piston problem in hyperelasticity with the stored energy in separable form
}

\author{
Draft version \\ Serge Ndanou ${ }^{a}$, Nicolas Favrie ${ }^{a}$ and Sergey Gavrilyuk ${ }^{a}$ \\ ${ }^{a}$ Aix-Marseille Université and C.N.R.S. U.M.R. 7343, IUSTI, 5 rue E. Fermi, \\ 13453 Marseille Cedex 13 France
}

\begin{abstract}
The piston problem for a hyperelastic hyperbolic conservative model where the stored energy is given in separable form is studied. The eigenfields corresponding to the hyperbolic system are of three types : linearly degenerate fields (corresponding to the contact characteristics), the fields which are genuinely nonlinear in the sense of Lax (corresponding to longitudinal waves), and, finally, nonlinear fields which are not genuinely nonlinear (corresponding to transverse waves). Taking the initial state free of stresses, we presented possible auto-similar solutions to the piston problem. In particular, we have shown that the equations admit transverse shock waves having a remarkable property : the solid density is decreasing through such a shock (it is a "rarefaction" shock).
\end{abstract}

\section{Résumé}

Problème du piston en hyperélasticité avec l'énergie interne sous forme séparable

Le problème du piston pour un modèle hyperélastique hyperbolique conservatif où l'énergie interne est donnée sous une forme séparée est étudié. Les champs caractéristiques associés aux valeurs propres et aux vecteurs propres du système hyperbolique sont de trois types : linéairement dégénéré (correspondant aux caractéristiques de contact), les champs qui sont vraiment nonlinéaires au sens de Lax (correspondant aux ondes longitudinales), et, enfin, les champs nonlinéaires qui ne sont pas vraiment nonlinéaires au sens de Lax (correspondant aux ondes transversales). Prenant l'état initial libre de contrainte, nous présentons de possible solutions auto-similaires du problème du piston. En particulier, nous avons montré que les équations admettent des ondes de choc transversales ayant une propriété remarquable : la densité du solide diminue à travers un tel choc (il s'agit d'une onde de choc de "raréfaction").

Key words: Nonlinear elasticity; piston problem; transverse waves

Mots-clés : Elasticité nonlinéaire; problème du piston; ondes transversales 


\section{Introduction}

An Eulerian hyperelastic hyperbolic conservative model is studied (G. H. Miller and P. Colella [1], S. K. Godunov and E. I. Romenskii [2] and others). We use here an equivalent formulation better adapted to the numerical study in the Eulerian coordinates (S.L. Gavrilyuk, N. Favrie, R. Saurel [3]). We consider the case of isotropic elastic solids where the stored energy is a function of the invariants of the Finger tensor (which is inverse to the left CauchyGreen tensor). The hyperelastic hyperbolic model can be extended to deal with visco-plasticity (Favrie et Gavrilyuk 2011) [4].Recently, we proposed a criterion of hyperbolicity of the equations of hyperelasticity in the case where the stored energy is taken in separable form : it is the sum of the energy depending only on the density and the entropy (hydrodynamic part), and the energy depending only on a the invariants of a reduced Finger tensor having unit determinant (isochoric part, or elastic part). In this paper, we study the piston problem for such a model. More exactly, we study auto-similar solutions appearing when the velocity is prescribed at the boundary of a non-deformed elastic half-space ("piston" problem). The fact that the elastic half-space is initially free of shear stresses allows us to simplify the solution. In particular, we constructed solutions containing transverse shocks in which the solid density after the shock is lower then that before the shock (so, this discontinuity is a "rarefaction" shock). This is a consequence of the fact that the eigenfields corresponding to transverse waves (shear waves) of the governing equations of hyperelasticity are not genuinely non-linear in the sense of Lax (E. Godlewski and P. - A. Raviart [5], P.D. Lax [6], P.G. LeFloch [7],D. Serre [8]).

The paper is organized as follows : in section 2 we present the governing equations and the hyperbolicity study; in section 3 we study the eigenfields; in section 4 the piston problem is solved.

\section{Governing Equations and Hyperbolicity}

\subsection{Governing equations of isotropic solids}

The general hyperelasticity model in the case of isotropic solids can be written as follows (G. H. Miller and P. Colella [1], S. K. Godunov and E. I. Romenskii

Email addresses: serge.ndanou@univ-amu.fr (Serge Ndanou), nicolas.favrie@univ-amu.fr (Nicolas Favrie), sergey.gavrilyuk@univ-amu.fr, corresponding author (Sergey Gavrilyuk). 
[2], S.L. Gavrilyuk, N. Favrie, R. Saurel [3]) :

$$
\left\{\begin{array}{l}
\frac{\partial \rho}{\partial t}+\operatorname{div}(\rho \mathbf{u})=0, \\
\frac{\partial(\rho \mathbf{u})}{\partial t}+\operatorname{div}(\rho \mathbf{u} \otimes \mathbf{u}-\boldsymbol{\sigma})=0 \\
\frac{\partial\left(\rho e+\frac{1}{2} \rho \mathbf{u}^{2}\right)}{\partial t}+\operatorname{div}\left(\left(\rho e+\frac{1}{2} \rho \mathbf{u}^{2}\right) \mathbf{u}-\boldsymbol{\sigma u}\right)=0 \\
\frac{\partial \mathbf{e}^{\beta}}{\partial t}+\nabla_{\mathbf{x}}\left(\mathbf{u} \cdot \mathbf{e}^{\beta}\right)=0, \quad \operatorname{rot}\left(\mathbf{e}^{\beta}\right)=0, \quad \beta=1,2,3 .
\end{array}\right.
$$

The operators div, rot and $\nabla$ are applied in the Eulerian coordinates $\mathbf{x}=$ $(x, y, z)^{T}$. Here $\rho$ is the solid density, $\mathbf{u}=(u, v, w)^{T}$ is the velocity field, $e(\mathbf{G}, \eta)$ is the internal energy, $\eta$ is the specific entropy, $\mathbf{G}=\left(\mathbf{F F}^{T}\right)^{-1}$ is the Finger tensor, $\mathbf{F}$ is the deformation gradient, $\boldsymbol{\sigma}$ is the Cauchy stress tensor defined as

$$
\boldsymbol{\sigma}=-2 \rho \frac{\partial e}{\partial \mathbf{G}} \mathbf{G}
$$

$\sigma$ is symmetric, because we deal with isotropic solids. The vectors $\mathbf{e}^{\beta}=$ $\left(a^{\beta}, b^{\beta}, c^{\beta}\right)^{T}$ are the columns of $\mathbf{F}^{-T}=\left(\mathbf{e}^{1}, \mathbf{e}^{2}, \mathbf{e}^{3}\right)$. Since $\mathbf{e}^{\beta}$ are gradients of the Lagrangian coordinates, necessarily, the compatibility condition is $\operatorname{rot}\left(\mathbf{e}^{\beta}\right)=$ 0 . This condition is time invariant : if it is satisfied initially, then it is satisfied for all the time.

We take the internal energy $e$ in separable form (R. J. Flory [9]) $: e=e^{h}(\rho, \eta)+$ $e^{e}(\mathbf{g})$, where $\eta$ is the specific entropy, $\rho=\rho_{0}|\mathbf{G}|^{\frac{1}{2}},|\mathbf{G}|=\operatorname{det}(\mathbf{G}), \rho_{0}$ is a reference density, $\mathbf{g}=\frac{\mathbf{G}}{|\mathbf{G}|^{\frac{1}{3}}}$ is a reduced Finger tensor. The stress tensor is also in separable form

$$
\boldsymbol{\sigma}=-p \mathbf{I}+\mathbf{S}, \quad p=\rho^{2} \frac{\partial e^{h}(\rho, \eta)}{\partial \rho}, \quad \mathbf{S}=-2 \rho \frac{\partial e^{e}}{\partial \mathbf{G}} \mathbf{G}, \quad \operatorname{tr}(\mathbf{S})=0
$$

The hydrodynamic sound speed $c$ is defined as

$$
c^{2}=\frac{\partial p}{\partial \rho}
$$

We will suppose that the following natural inequalities are satisfied :

$$
\frac{\partial p}{\partial \rho}>0, \frac{\partial p}{\partial \eta}>0
$$

The following particular forms of the energy can be used in applications :

$$
e^{h}(\rho, \eta)=\frac{\operatorname{Aexp}\left(\frac{\eta-\eta_{0}}{c_{v}}\right) \rho^{\gamma}+(\gamma-1) p_{\infty}}{(\gamma-1) \rho},
$$




$$
e^{e}(\mathbf{g})=\frac{\mu}{8 \rho_{0}}\left(\operatorname{tr}\left(\mathbf{g}^{2}\right)-3\right) .
$$

The coefficients $A, \eta_{0}, p_{\infty}, \gamma>1, c_{v}, \mu$ are constant. In the limit of small deformations these equations give us Hooke's law. Obviously, the hydrodynamic part of the energy verifies inequalities (4).

A non-conservative form of (1) is :

$$
\left\{\begin{array}{l}
\frac{\partial \rho}{\partial t}+\mathbf{u} \cdot \nabla \rho+\rho \operatorname{div}(\mathbf{u})=0 \\
\frac{\partial \mathbf{u}}{\partial t}+\frac{\partial \mathbf{u}}{\partial \mathbf{x}} \mathbf{u}+\frac{\nabla p}{\rho}-\frac{\operatorname{div}(\mathbf{S})}{\rho}=0 \\
\frac{\partial \eta}{\partial t}+\mathbf{u} \cdot \nabla \eta=0 \\
\frac{\partial \mathbf{e}^{\beta}}{\partial t}+\frac{\partial \mathbf{e}^{\beta}}{\partial \mathbf{x}} \mathbf{u}+\left(\frac{\partial \mathbf{u}}{\partial \mathbf{x}}\right)^{T} \mathbf{e}^{\beta}=0, \quad \beta=1,2,3
\end{array}\right.
$$

These equations are invariant under the transformation group

$$
t^{\prime}=t, \quad \mathbf{x}^{\prime}=O \mathbf{x}, \quad \mathbf{u}^{\prime}=O \mathbf{u}, \quad \mathbf{e}^{\beta \prime}=O \mathbf{e}^{\beta}, \quad \rho^{\prime}=\rho, \quad \eta^{\prime}=\eta,
$$

where $O$ is any element of $S O(3)$. In particular, it allows us to reduce the hyperbolicity study of the multi-dimensional system (7) to the hyperbolicity study of the corresponding 1D system (see for detail S. Ndanou, N. Favrie and S. Gavrilyuk [10]. In the case of (5) and (6), the equations (7) are hyperbolic for any deformations. In particular, this will imply the hyperbolicity of the system (1) because the system (7) contains (1) in a particular case where $\operatorname{rot}\left(\mathbf{e}^{\beta}\right)=0$. The proof of hyperbolicity of (7) is based on the following technical Lemma.

Lemma 2.1 (S. Ndanou, N. Favrie and S. Gavrilyuk [10]). Let the energy $e^{e}(\mathbf{g})$ be an isotropic function of $\mathbf{g}=\frac{\mathbf{G}}{|\mathbf{G}|^{\frac{1}{3}}}$, i.e. $e^{e}(\mathbf{g})=e^{e}\left(j_{1}, j_{2}\right)$, where $j_{i}=\operatorname{tr}\left(\mathbf{g}^{i}\right), i=1,2$. Let us introduce the vectors $\mathbf{a}=\left(a^{\alpha}\right), \mathbf{b}=\left(b^{\alpha}\right)$ and $\mathbf{c}=\left(c^{\alpha}\right), \alpha=1,2,3$. Then the deviatoric part of the stress tensor can be expressed as :

$$
\mathbf{S}=-2 \rho \frac{\partial e^{e}}{\partial \mathbf{G}} \mathbf{G}=-\rho\left(\begin{array}{lll}
\frac{\partial e^{e}}{\partial \mathbf{a}} \mathbf{a} & \frac{\partial e^{e}}{\partial \mathbf{a}} \mathbf{b} & \frac{\partial e^{e}}{\partial \mathbf{a}} \mathbf{c} \\
\frac{\partial e^{e}}{\partial \mathbf{a}} \mathbf{b} & \frac{\partial e^{e}}{\partial \mathbf{b}} \mathbf{b} & \frac{\partial e^{e}}{\partial \mathbf{c}} \mathbf{b} \\
\frac{\partial e^{e}}{\partial \mathbf{a}} \mathbf{c} & \frac{\partial e^{e}}{\partial \mathbf{c}} \mathbf{b} & \frac{\partial e^{e}}{\partial \mathbf{c}} \mathbf{c}
\end{array}\right)
$$

Moreover,

$$
\frac{\partial e^{e}}{\partial \mathbf{a}} \mathbf{b}=\frac{\partial e^{e}}{\partial \mathbf{b}} \mathbf{a}, \quad \frac{\partial e^{e}}{\partial \mathbf{a}} \mathbf{c}=\frac{\partial e^{e}}{\partial \mathbf{c}} \mathbf{a}, \quad \frac{\partial e^{e}}{\partial \mathbf{b}} \mathbf{c}=\frac{\partial e^{e}}{\partial \mathbf{c}} \mathbf{b}
$$

In particular, the lemma 2.1 guarantees the symmetry of $\mathbf{S}$. 
Consider the 1D case where all the variables depend only on $(t, x)$. Moreover, $\mathbf{u}=(u, v, w)=(u, v, 0), a^{3}=0, b^{1}=0, b^{2}=1, b^{3}=0, c^{1}=0, c^{2}=0, c^{3}=1$.

The corresponding system of equations is as follows :

$$
\left\{\begin{array}{l}
\frac{\partial \rho}{\partial t}+u \frac{\partial \rho}{\partial x}+\rho \frac{\partial u}{\partial x}=0 \\
\frac{\partial a^{1}}{\partial t}+u \frac{\partial a^{1}}{\partial x}+a^{1} \frac{\partial u}{\partial x}=0 \\
\frac{\partial a^{2}}{\partial t}+u \frac{\partial a^{2}}{\partial x}+a^{2} \frac{\partial u}{\partial x}+\frac{\partial v}{\partial x}=0 \\
\frac{\partial u}{\partial t}+u \frac{\partial u}{\partial x}+\left(\frac{c^{2}}{\rho}-\frac{1}{\rho} \frac{\partial S_{11}}{\partial \rho}\right) \frac{\partial \rho}{\partial x}-\frac{1}{\rho} \frac{\partial S_{11}}{\partial a^{1}} \frac{\partial a^{1}}{\partial x}-\frac{1}{\rho} \frac{\partial S_{11}}{\partial a^{2}} \frac{\partial a^{2}}{\partial x}+\frac{\partial p}{\partial \eta} \frac{\partial \eta}{\partial x}=0 \\
\frac{\partial v}{\partial t}+u \frac{\partial v}{\partial x}-\frac{1}{\rho} \frac{\partial S_{12}}{\partial \rho} \frac{\partial \rho}{\partial x}-\frac{1}{\rho} \frac{\partial S_{12}}{\partial a^{1}} \frac{\partial a^{1}}{\partial x}-\frac{1}{\rho} \frac{\partial S_{12}}{\partial a^{2}} \frac{\partial a^{2}}{\partial x}=0 \\
\frac{\partial \eta}{\partial t}+u \frac{\partial \eta}{\partial x}=0
\end{array}\right.
$$

If we set $\mathbf{U}=\left(\rho, a^{1}, a^{2}, u, v, \eta\right)^{T}$, the system can be written as follows

$$
\frac{\partial \mathbf{U}}{\partial t}+\mathbf{A} \frac{\partial \mathbf{U}}{\partial x}=0
$$

with

$$
\mathbf{A}=\left(\begin{array}{cccccc}
u & 0 & 0 & \rho & 0 & 0 \\
0 & u & 0 & a^{1} & 0 & 0 \\
0 & 0 & u & a^{2} & 1 & 0 \\
\frac{c^{2}}{\rho}-\frac{1}{\rho} \frac{\partial S_{11}}{\partial \rho} & -\frac{1}{\rho} \frac{\partial S_{11}}{\partial a^{1}} & -\frac{1}{\rho} \frac{\partial S_{11}}{\partial a^{2}} & u & 0 & \frac{\partial p}{\partial \eta} \\
-\frac{1}{\rho} \frac{\partial S_{12}}{\partial \rho} & -\frac{1}{\rho} \frac{\partial S_{12}}{\partial a^{1}} & -\frac{1}{\rho} \frac{\partial S_{12}}{\partial a^{2}} & 0 & u & 0 \\
0 & 0 & 0 & 0 & 0 & u
\end{array}\right)
$$

Lemma 2.2 The eigenvalues $\nu_{6}>\nu_{5}>v_{4}=\nu_{3}>\nu_{2}>\nu_{1}$ of the matrix $A$ are given by:

$$
\begin{gathered}
\nu_{1,6}=u \pm \sqrt{\frac{\operatorname{tr}(\mathbf{K})+\sqrt{\Delta}}{2}}, \\
\nu_{2,5}=u \pm \sqrt{\frac{\operatorname{tr}(\mathbf{K})-\sqrt{\Delta}}{2}}, \\
\nu_{3,4}=u,
\end{gathered}
$$

where

$$
\mathbf{K}=\left(\begin{array}{ll}
c^{2} & 0 \\
0 & 0
\end{array}\right)+\mathbf{M}
$$




$$
\begin{gathered}
\mathbf{M}=\left(\begin{array}{cc}
\frac{\partial e^{e}}{\partial \mathbf{a}} \cdot \mathbf{a}+\frac{\partial}{\partial \mathbf{a}}\left(\frac{\partial e^{e}}{\partial \mathbf{a}} \cdot \mathbf{a}\right) \cdot \mathbf{a} & \frac{\partial e^{e}}{\partial \mathbf{a}} \cdot \mathbf{b}+\frac{\partial}{\partial \mathbf{a}}\left(\frac{\partial e^{e}}{\partial \mathbf{a}} \cdot \mathbf{b}\right) \cdot \mathbf{a} \\
\frac{\partial e^{e}}{\partial \mathbf{a}} \cdot \mathbf{b}+\frac{\partial}{\partial \mathbf{a}}\left(\frac{\partial e^{e}}{\partial \mathbf{a}} \cdot \mathbf{b}\right) \cdot \mathbf{a} & \frac{\partial}{\partial \mathbf{a}}\left(\frac{\partial e^{e}}{\partial \mathbf{a}} \cdot \mathbf{b}\right) \cdot \mathbf{b}
\end{array}\right), \\
\Delta=(\operatorname{tr}(\mathbf{K}))^{2}-4 \operatorname{det}(\mathbf{K})=\left(K_{11}-K_{22}\right)^{2}+4 K_{12}^{2}, \\
a=\left(a^{1}, a^{2}\right) \text { and } b=(0,1) .
\end{gathered}
$$

The proof of the Lemma 2.2 is direct. The Lemma 2.1 was used to obtain a symmetric form of $\mathbf{K}$.

Note that $\mathbf{M}$ is positive definite for the equation of state (6) (S. Ndanou, N. Favrie and S. Gavrilyuk [10]). Since $c^{2}>0, \mathbf{K}$ is also positive definite. In particular, the eigenvalues $\nu_{i}, i=1, \ldots, 6$ are real. We find now the corresponding right eigenvectors of $\mathbf{A}$.

The eigenvectors $\mathbf{V}_{3}=(1,0,0,0,0,0)^{T}$ and $\mathbf{V}_{4}=(0,0,0,0,0,1)^{T}$ correspond to the eigenvalues $\nu_{3}=\nu_{4}=u$. For the eigenvalues $\nu_{i}, i=1,6$ corresponding to longitudinal waves the eigenvectors are :

$$
\mathbf{V}_{i}=\left(\frac{\rho}{\left(\nu_{i}-u\right)}, \frac{a^{1}}{\left(\nu_{i}-u\right)}, \frac{2 a^{2} K_{12}+K_{22}-K_{11}+\sqrt{\Delta}}{2 K_{12}\left(\nu_{i}-u\right)}, 1, \frac{K_{22}-K_{11}+\sqrt{\Delta}}{2 K_{12}}, 0\right) .
$$

Since

$$
\left(K_{22}-K_{11}\right)+\sqrt{\Delta}=\frac{\left(K_{22}-K_{11}\right)^{2}-\Delta}{\left(K_{22}-K_{11}\right)-\sqrt{\Delta}}=\frac{-4 K_{12}^{2}}{\left(K_{22}-K_{11}\right)+\sqrt{\Delta}} .
$$

the eigenvectors are not singular when $K_{12}$ vanishes.

For the eigenvalues $\nu_{i}, i=2,5$ corresponding to transverse waves the eigenvectors are :

$$
\mathbf{V}_{i}=\left(\frac{\rho}{\left(\nu_{i}-u\right)}, \frac{a^{1}}{\left(\nu_{i}-u\right)}, \frac{2 a^{2} K_{12}+K_{22}-K_{11}-\sqrt{\Delta}}{2 K_{12}\left(\nu_{i}-u\right)}, 1, \frac{K_{22}-K_{11}-\sqrt{\Delta}}{2 K_{12}}, 0\right) .
$$

The eigenvalues can be ordered :

$$
\nu_{1}>\nu_{2}>\nu_{3}=\nu_{4}>\nu_{5}>\nu_{6}
$$

$\nu_{1}$ and $\nu_{6}$ are longitudinal wave speeds, $\nu_{2}$ and $\nu_{5}$ are transverse wave speeds, and $\nu_{3}$ or $\nu_{4}$ are speeds of contact characteristics. Let us also remark that the equation of the density can be integrated in the form

$$
\rho=\rho_{0} a^{1} .
$$

Here $\rho_{0}$ is a constant. In general, it can be a function which is conserved along trajectories. 


\section{$3 \quad$ Study of eigenfields}

\subsection{Eigenfields associated to $\nu_{3}=\nu_{4}=u$}

Obviously, these fields are linearly degenerate $: \nabla \nu_{3} . \mathbf{V}_{3}=\nabla \nu_{4} \cdot \mathbf{V}_{4}=0$.

\subsection{Eigenfields associated to $\nu_{1}$ and $\nu_{6}$}

These fields corresponding to longitudinal waves and estimated on the variety (12) are genuinely non-linear in the sense of Lax :

$$
\left.\nabla \nu_{1} \cdot \mathbf{V}_{1}\right|_{\rho=\rho_{0} a^{1}}>0
$$

This result can easily be checked analytically in the vicinity of the equilibrium $\left(a^{1}=1, a^{2}=0\right)$, and numerically out of equilibrium.

\subsubsection{Simple waves}

We are looking for the solution of (10) in the form :

$$
\mathbf{U}(t, x)=\mathbf{U}\left(\frac{x}{t}\right)=\mathbf{U}(\xi)
$$

Then $\mathbf{U}$ verifies the following system :

$$
(\mathbf{A}-\xi \mathbf{I}) \frac{d \mathbf{U}}{d \xi}=0 .
$$

Hence, $\xi$ is an eigenvalue of $\mathbf{A}$, and $\frac{d \mathbf{U}}{d \xi}$ is the corresponding right eigenvector. In particular, for the field $\nu_{1}$ one has

$$
\frac{d \mathbf{U}}{d \xi}=\frac{\mathbf{V}_{1}}{\nabla \nu_{1} \cdot \mathbf{V}_{1}} .
$$

Or, in developed form :

$$
\begin{aligned}
\frac{d \rho}{d \xi} & =\frac{\rho}{\left(\nu_{1}-u\right) \nabla \nu_{1} \cdot \mathbf{V}_{1}} \\
\frac{d a^{1}}{d \xi} & =\frac{a^{1}}{\left(\nu_{1}-u\right) \nabla \nu_{1} \cdot \mathbf{V}_{1}},
\end{aligned}
$$




$$
\frac{d a^{2}}{d \xi}=\frac{2 a^{2} K_{12}+K_{22}-K_{11}+\sqrt{\Delta}}{2 K_{12}\left(\nu_{1}-u\right) \nabla \nu_{1} \cdot \mathbf{V}_{1}}=\frac{a^{2}}{\left(\nu_{1}-u\right) \nabla \nu_{1} \cdot \mathbf{V}_{1}}+\frac{2 K_{12}}{\left.\left(K_{11}-K_{22}\right)+\sqrt{\Delta}\right)\left(\nu_{1}-u\right) \nabla \nu_{1} \cdot \mathbf{V}_{1}}
$$

$$
\begin{gathered}
\frac{d u}{d \xi}=\frac{1}{\nabla \nu_{1} \cdot \mathbf{V}_{1}}, \\
\frac{d v}{d \xi}=\frac{K_{22}-K_{11}+\sqrt{\Delta}}{2 K_{12} \nabla \nu_{1} \cdot \mathbf{V}_{1}}, \\
\frac{d \eta}{d \xi}=0 .
\end{gathered}
$$

One can prove that in the case

$$
e^{e}(\mathbf{g})=\frac{\mu}{8 \rho_{0}}\left(j_{2}-3\right),
$$

$K_{12}$ is proportional to $a^{2}$. Indeed,

$$
\begin{gathered}
K_{12}=\frac{\mu}{8 \rho_{0}}\left(\frac{\partial j_{2}}{\partial \mathbf{a}} \cdot \mathbf{b}+\frac{\partial}{\partial \mathbf{a}}\left(\frac{\partial j_{2}}{\partial \mathbf{a}} \cdot \mathbf{b}\right) \cdot \mathbf{a}\right) \\
=\frac{\mu}{8 \rho_{0}}\left(\frac{4 a^{2}\left(\left(a^{1}\right)^{2}+\left(a^{2}\right)^{2}+1\right)}{\left(a^{1}\right)^{4 / 3}}+a^{1} \frac{\partial}{\partial a^{1}}\left(\frac{4 a^{2}\left(\left(a^{1}\right)^{2}+\left(a^{2}\right)^{2}+1\right)}{\left(a^{1}\right)^{4 / 3}}\right)\right. \\
\left.+a^{2} \frac{\partial}{\partial a^{2}}\left(\frac{4 a^{2}\left(\left(a^{1}\right)^{2}+\left(a^{2}\right)^{2}+1\right)}{\left(a^{1}\right)^{4 / 3}}\right)\right) .
\end{gathered}
$$

Hence, if initially $\left(a^{2}\right)$ was zero, it will stay zero. The equation for the vertical velocity $v$ also gives the solution $v=0$ if it was initially zero. In particular, the equations admit the following Riemann invariant corresponding to the right facing waves where we have to replace $\rho=\rho_{0} a^{1}$ :

$$
u-\int^{a^{1}} \frac{\sqrt{\frac{\operatorname{tr}(\mathbf{K})+\sqrt{\Delta}}{2}}}{a^{1}} d a^{1}=\text { const. }
$$

The study of the field $\nu_{6}$ gives another invariant corresponding to the left facing waves :

Since

$$
u+\int^{a^{1}} \frac{\sqrt{\frac{\operatorname{tr}(\mathbf{K})+\sqrt{\Delta}}{2}}}{a^{1}} d a^{1}=\text { const. }
$$

$$
\left.\nabla \nu_{1,6} \cdot \mathbf{V}_{1,6}\right|_{\rho=\rho_{0} a^{1}}>0
$$

the longitudinal simple waves are always rarefaction waves (in which the density decreases). These invariants are reminiscent of those for the Euler equations of compressible fluids. 


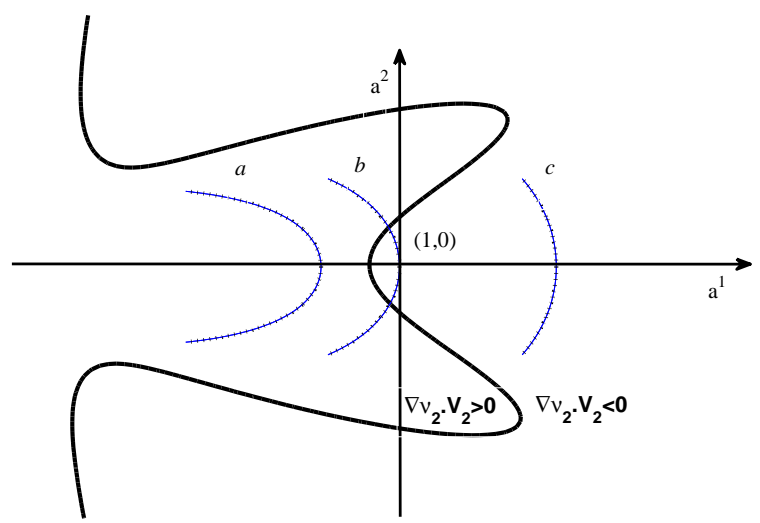

Figure 1. The curve where $\left.\nabla \nu_{2} \cdot \mathbf{V}_{2}\right|_{\rho=\rho_{0} a^{1}}=0$ is shown in bold line. Different invariant curves associated to the eigenfield $\nu_{2}$ are shown by dashed lines.

\subsection{Eigenfields associated to $\nu_{2}$ or $\nu_{5}$}

These eigenvalues correspond to the transverse waves. Obviously, $\nabla \nu_{2} \cdot \mathbf{V}_{2}=$ $-\nabla \nu_{5} . V_{5}$. Hence, it is sufficient to study only the field $\nu_{2}$. The Figure 1 shows that these fields estimated at the variety $\rho=\rho_{0} a^{1}$ are not genuinely nonlinear in the sense of Lax (P.G. LeFloch [7] for the solution of the Riemann problem in the case of such eigenfields). The curve where $\left.\nabla \nu_{2} \cdot \mathbf{V}_{2}\right|_{\rho=\rho_{0} a^{1}}$ vanishes is shown in bold line.

\subsubsection{Simple waves}

The equations corresponding to the eigenvalue $\nu_{2}$ are :

$$
\begin{gathered}
\frac{d \rho}{d \xi}=\frac{\rho}{\left(\nu_{2}-u\right)\left(\nabla \nu_{2} \cdot \mathbf{V}_{2}\right)} \\
\frac{d a^{1}}{d \xi}=\frac{a^{1}}{\left(\nu_{2}-u\right)\left(\nabla \nu_{2} \cdot \mathbf{V}_{2}\right)}, \\
\frac{d a^{2}}{d \xi}=\frac{2 a^{2} K_{12}+K_{22}-K_{11}-\sqrt{\Delta}}{2 K_{12}\left(\nu_{2}-u\right)\left(\nabla \nu_{2} \cdot \mathbf{V}_{2}\right)} \\
\frac{d u}{d \xi}=\frac{1}{\left(\boldsymbol{\nabla} \nu_{2} \cdot \mathbf{V}_{2}\right)} \\
\frac{d v}{d \xi}=\frac{K_{22}-K_{11}-\sqrt{\Delta}}{2 K_{12}\left(\nabla \nu_{2} \cdot \mathbf{V}_{2}\right)} \\
\frac{d s}{d \xi}=0 .
\end{gathered}
$$


$\mathbf{V}_{i}=\left(\frac{\rho}{\left(\nu_{i}-u\right)}, \frac{a^{1}}{\left(\nu_{i}-u\right)}, \frac{2 a^{2} K_{12}+K_{22}-K_{11}-\sqrt{\Delta}}{2 K_{12}\left(\nu_{i}-u\right)}, 1, \frac{K_{22}-K_{11}-\sqrt{\Delta}}{2 K_{12}}, 0\right)$

The Riemann invariant for the right facing transverse waves (corresponding to $\left.\nu_{2}\right)$ is

$$
u-\int^{a^{1}} \frac{\sqrt{\frac{\operatorname{tr}(\mathbf{K})-\sqrt{\Delta}}{2}}}{a^{1}} d a^{1}=\text { const. }
$$

For for the left facing transverse waves the corresponding Riemann invariant is :

$$
u+\int^{a^{1}} \frac{\sqrt{\frac{\operatorname{tr}(\mathbf{K})-\sqrt{\Delta}}{2}}}{a^{1}} d a^{1}=\text { const }
$$

In these formulas $a^{2}$ should be replaced as a function of $a^{1}$ as the solution of the following Cauchy problem (always calculated for $\rho=\rho_{0} a^{1}$ ):

$$
\begin{gathered}
\frac{d a^{2}}{d a^{1}}=\frac{a^{2}}{a^{1}}+\frac{2 K_{12}}{a^{1}\left(K_{11}-K_{22}+\sqrt{\Delta}\right)}, \\
\left.a^{2}\right|_{a^{1}=a_{*}^{1}}=0 .
\end{gathered}
$$

Here $a_{*}^{1}$ is a state obtained from the state $a^{1}$ by a simple longitudinal wave which is always rarefaction wave, or by the longitudinal shock (see the discussion below about Rankine- Hugoniot relations). In the vicinity of the state $\left(a^{1}=1, a^{2}=0\right)$ the expression $\left.\nabla \nu_{2} \cdot \mathbf{V}_{2}\right|_{\rho=\rho_{0} a^{1}}$ is negative. Hence, if the longitudinal rarefaction waves (where $a^{1}$ is decreasing) are not too strong (i.e. $\nabla \nu_{2} .\left.\mathbf{V}_{2}\right|_{\rho=\rho_{0} a^{1 *}}$ is negative at $\left.\left(a_{*}^{1}, a^{2}=0\right)\right)$, the eigenvalue $\nu_{2}$ has a minimum along each simple wave passing by that point. Hence, the simple transverse waves does not exist in this case. In the case of strong longitudinal rarefaction waves transforming the state $\left(a^{1}=1, a^{2}=0\right)$ into $\left(a_{*}^{1}<1, a^{2}=0\right)$ where $\nabla \nu_{2} .\left.\mathbf{V}_{2}\right|_{\rho=\rho_{0} a^{1} *}$ is positive (a curve $a$ in Figure 1 ) one can have simple transverse waves which are also rarefaction waves (the density will decrease).

\section{Rankine-Hugoniot relations}

For any variable $X$, we denote $[X]=X^{r}-X^{l}$ (the difference between right and left values at the discontinuity). The Rankine-Hugoniot relations coming from the conservative system (1) in the case where $a^{3}=0, \quad w=0, \quad b^{1}=$ 


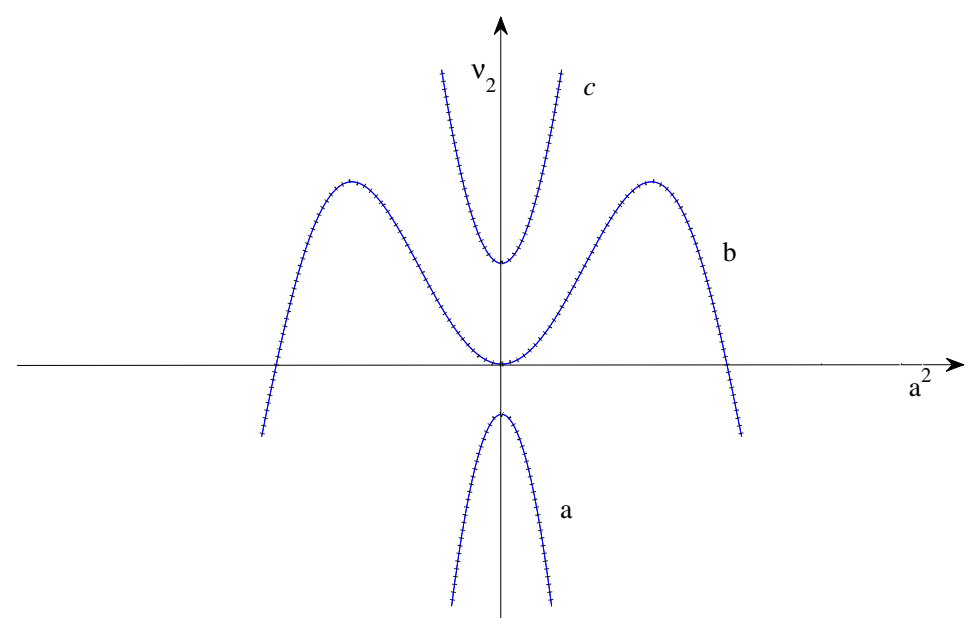

FiguRE 2. The behavior of characteristic speed $\nu_{2}$ along transverse waves is shown. The first case (a) corresponds to the rarefaction curve containing in the domain $\nabla \nu_{2} .\left.\mathbf{V}_{2}\right|_{\rho=\rho_{0} a^{1}}>0$ (see Figure 1). The transverse rarefaction waves are thus possible. In the cases (b) and (c) the continuous rarefaction waves do not exist.

$0, \quad b^{2}=1, \quad b^{3}=0, \quad c^{1}=0, \quad c^{2}=0, \quad c^{3}=1$, can be written as :

$$
\left\{\begin{array}{l}
{[\rho(u-D)]=[m]=0} \\
m[u]=\left[\sigma_{11}\right], \\
m[v]=\left[\sigma_{12}\right], \\
m\left[e+\frac{1}{2}\left(u^{2}+v^{2}\right)\right]=\left[\sigma_{11} u+\sigma_{12} v\right], \\
m\left[\frac{a^{1}}{\rho}\right]=0, \\
m\left[\frac{a^{2}}{\rho}\right]+[v]=0 .
\end{array}\right.
$$


Here we denoted $m=\rho(u-D)$ where $D$ is the velocity of the discontinuity. They are equivalent to :

$$
\left\{\begin{array}{l}
{[\rho(u-D)]=0} \\
m[u]=\left[\sigma_{11}\right], \\
m[v]=\left[\sigma_{12}\right], \\
m[e]=m \frac{\sigma_{11}^{l}+\sigma_{11}^{r}}{2}[\tau]-m \frac{\sigma_{12}^{r}+\sigma_{12}^{l}}{2}\left[a^{2} \tau\right], \\
m\left[\frac{a^{1}}{\rho}\right]=0, \\
m\left[\frac{a^{2}}{\rho}\right]+[v]=0,
\end{array}\right.
$$

where $\tau=1 / \rho$ is the specific volume.

Through the contact discontinuities where $m=0$ we get $u_{r}=D=u_{l}$, $\left[\sigma_{11}\right]=0,\left[\sigma_{12}\right]=0$ and $[v]=0$. For shocks where $m \neq 0$, we will distinguish two types of shocks : longitudinal and transverse ones.

\subsection{Longitudinal shock waves}

We suppose in this part that $m \neq 0$. Obviously, $[v]=0 \Leftrightarrow\left[\sigma_{12}\right]=0 \Leftrightarrow$ $\left[a^{2} \tau\right]=0, \quad \tau=\frac{1}{\rho}$. Longitudinal shock waves are defined by $\left[a^{2} \tau\right]=0$. The Rankine-Hugoniot relations for longitudinal waves are written as :

$$
\left\{\begin{array}{l}
D=\frac{u_{r} \rho_{r}-u_{l} \rho_{l}}{\rho_{r}-\rho_{l}}, \\
{\left[a^{2} \tau\right]=0,} \\
{[u]^{2}=\left[\sigma_{11}\right][\tau],} \\
{[e]=\frac{\sigma_{11}^{l}+\sigma_{11}^{r}}{2}[\tau] .} \\
{[v]=0,} \\
{\left[\sigma_{12}\right]=0 .}
\end{array}\right.
$$

$a^{2}$ vanishes after the shock if it was zero before the shock. The same statement is valid for the transversal velocity $v$. 


\subsection{Transverse shock waves}

Consider now transverse shock waves. In this case $\left[a^{2} \tau\right] \neq 0$ This give us :

$$
\left\{\begin{array}{l}
D=\frac{u_{r} \rho_{r}-u_{l} \rho_{l}}{\rho_{r}-\rho_{l}} \\
m[u]=\left[\sigma_{11}\right] \\
m[v]=\left[\sigma_{12}\right], \\
{[e]=\frac{\sigma_{11}^{l}+\sigma_{11}^{r}}{2}[\tau]-\frac{\sigma_{12}^{r}+\sigma_{12}^{l}}{2}\left[a^{2} \tau\right]} \\
{\left[\frac{a^{1}}{\rho}\right]=0,} \\
m\left[a^{2} \tau\right]+[v]=0
\end{array}\right.
$$

Since $[u]=m[\tau], m[u]=\left[\sigma_{11}\right], m^{2}[\tau]=\left[\sigma_{11}\right]$ and $m^{2}[v]^{2}=\left[\sigma_{12}\right]^{2}$, we finally get :

$$
\left\{\begin{array}{l}
D=\frac{u_{r} \rho_{r}-u_{l} \rho_{l}}{\rho_{r}-\rho_{l}}, \\
m[u]=\left[\sigma_{11}\right], \\
m[v]=\left[\sigma_{12}\right], \\
{[e]=\frac{\sigma_{11}^{l}+\sigma_{11}^{r}}{2}[\tau]-\frac{\sigma_{12}^{r}+\sigma_{12}^{l}}{2}\left[a^{2} \tau\right],} \\
{[u]^{2}=\left[\sigma_{11}\right][\tau],} \\
{[v]^{2}=\frac{\left[\sigma_{12}\right]^{2}[\tau]}{\left[\sigma_{11}\right]},} \\
{\left[\frac{a^{1}}{\rho}\right]=0,} \\
{\left[\sigma_{11}\right]\left[a^{2} \tau\right]+\left[\sigma_{12}\right][\tau]=0 .}
\end{array}\right.
$$

\section{The piston problem}

\subsection{A special piston problem}

Consider a piston (an infinite plane described initially as $x=0$ ) which is sticked to an elastic solid at rest situated at $x>0$. Initially, the elastic solid is free of shear stresses. The variables in this state will be denoted by index "0". 

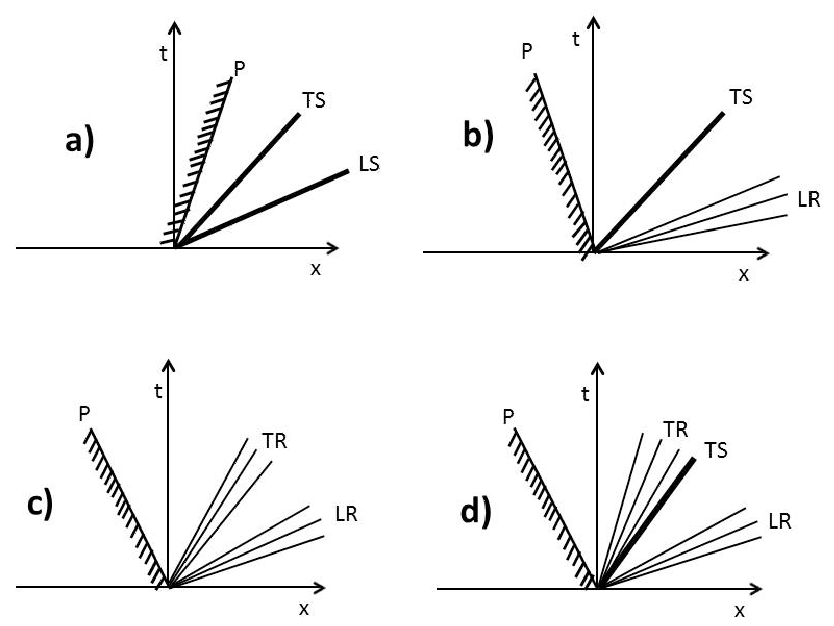

FiguRE 3. We have four different general configurations for the piston problem where the initial state is in equilibrium : The first case : a longitudinal shock wave (LS) is followed by a transverse shock wave (TS). This configuration can appear not only in the case where the horizontal piston velocity is positive. The other three cases can appear only if the horizontal piston velocity is negative. The second case : a longitudinal rarefaction wave (LR) is followed by a transverse shock wave (TS). The third case : a longitudinal rarefaction wave (LR) is followed by a transverse rarefaction wave (TR). The fourth case : a longitudinal rarefaction wave (LR) is followed by a transverse characteristic shock wave (TS) to which a transverse rarefaction wave (TR) is sticked.

So, $a_{0}^{1}=1, a_{0}^{2}=0, u_{0}=v_{0}=0$. The piston becomes to move at time $t=0$ with a given velocity $\left(u_{p}, v_{p}\right)$. In the case of $v_{p}=0$ the solution is simple. If $u_{p}>0$ then we have a longitudinal shock wave. If $u_{p}<0$, the solution is the longitudinal rarefaction wave. Consider the case where $u_{p}>0$ and $v_{p}$ takes any value (not too large to stay in the domain where $\left.\nabla \nu_{2} \cdot \mathbf{V}_{2}\right|_{\rho=\rho_{0} a^{1}}<0$ ). The state " 0 " will be transformed by a longitudinal shock wave into the state "*" where $a_{*}^{1}>1, a_{*}^{2}=a_{0}^{2}=0$ following by a transverse shock (see Figure 3, the first case). The transverse shock is always a rarefaction shock (i.e. the solid density will decrease after the transverse shock ). In the Figure the classical different configurations that we can have.

However, in the case where $u_{p}$ is negative, the solution depends on the value of $v_{p}$. One can have several different situations. The longitudinal rarefaction wave $(L R)$ transforms the state "0" into a state "*" where we have always $\left.\nabla \nu_{2} \cdot \mathbf{V}_{2}\right|_{\rho=\rho_{0} a^{1} *}<0$. Then it will followed by the transverse shock TS (the second case in Figure 3). The transverse wave can also be a characteristic shock, i. e. the wave can be followed by a rarefaction continuous transverse wave (the fourth case). Very large longitudinal rarefaction waves can also be followed by a transverse rarefaction wave $(R T)$. 


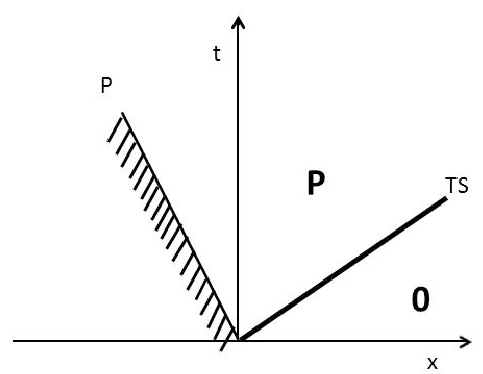

FiguRE 4. Solution with a unique transverse wave

We ask now the following question : what is the relation between $u_{p}$ and $v_{p}$ allowing us to have a solution containing only one transverse shock relating the state " 0 " to a state " $\mathrm{P}$ " where the velocity is prescribed $:(u, v)_{p}=\left(u_{p}, v_{p}\right)$ (see Figure 4). In a sense, this limiting curve will separate the the first basic configuration $L S \rightarrow T S$ (which can happen also for negative horizontal piston velocities when the vertical velocities are quite large), from the other three configurations (see Figure 3). This degenerate configuration is shown in Figure 5 . We wish to connect the equilibrium state "0" with a state "P" by transverse

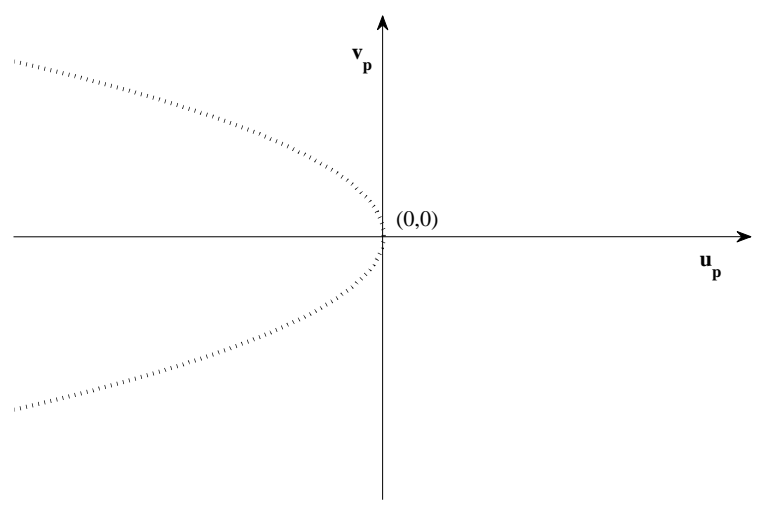

FIGURE 5 . When the piston velocity takes the values belonging to the limit curve shown by dashed line, the only solution is the $T S$ wave. Outside this curve we have a two-wave configuration $L S \rightarrow T S$. Inside this curve, the three other configurations can occur.

shock wave. The Rankine-Hugoniot relations are :

$$
\left(u_{P}\right)^{2}=\left(\left(\sigma_{11}\right)_{P}-\left(\sigma_{11}\right)_{0}\right)\left(\tau_{P}-\tau_{0}\right),
$$




$$
\begin{gathered}
\left(v_{P}\right)^{2}=\frac{\left(\left(\sigma_{12}\right)_{P}\right)^{2}}{\left(\left(\sigma_{11}\right)_{P}-\left(\sigma_{11}\right)_{0}\right)}\left(\tau_{P}-\tau_{0}\right), \\
0=\left(\left(\sigma_{12}\right)_{P}\right)\left(\tau_{P}-\tau_{0}\right)+\left(\left(a^{2} \tau\right)_{P}\right)\left(\left(\sigma_{11}\right)_{P}-\left(\sigma_{11}\right)_{0}\right), \\
\left(e_{P}-e_{0}\right)=\frac{1}{2}\left(\left(\sigma_{11}\right)_{P}+\left(\sigma_{11}\right)_{0}\right)\left(\tau_{P}-\tau_{0}\right)+\frac{1}{2}\left(\left(\sigma_{12}\right)_{P}\right)\left(\left(a^{2} \tau\right)_{P}\right) .
\end{gathered}
$$

We have four relations for three unknowns $a_{P}^{1}, a_{P}^{2}$ and $\eta_{P}$. Hence, it allows us to relate the parameters $u_{P}$ and $v_{P}$.

\section{CONCLUSION}

The piston problem for a hyperelastic hyperbolic conservative model where the stored energy is given in separable form is studied. Such an exact solution is very useful to evaluate the convergence of numerical schemes. The eigenfields corresponding to the hyperbolic system are of three types : linearly degenerate fields (corresponding to the contact characteristics), the fields which are genuinely nonlinear in the sense of Lax (corresponding to longitudinal waves), and nonlinear fields which are not genuinely nonlinear (corresponding to transverse waves). Taking the initial state free of stresses, we presented possible autosimilar solutions to the piston problem. In particular, we have shown that the equations admit transverse shock waves having a remarkable property : the density is decreasing through such a shock.

For numerical applications, the solution of the general Riemann problem is needed. However, such a problem is much more complicated because its solution depends on the choice of the equations of state (see, for example, A. Kulikovskii and E. Sveshnikova [11] for the study of the Riemann problem for a polynomial equation of state).

\section{Références}

[1] G. H. Miller and P. Colella, A high order Eulerian Godunov method for elastic plastic flow in solids, Journal of Computational Physics, 167, 131-176, 2001.

[2] S.K. Godunov, E. I. Romenskii, Elements of Continuum Mechanics and Conservation Laws, Kluwer Academic Publishers, N. Y., 2003.

[3] S. L. Gavrilyuk, N. Favrie \& R. Saurel, Modeling wave dynamics of compressible elastic materials, Journal of Computational Physics, 227, 2941-2969, 2008.

[4] N. Favrie \& S.Gavrilyuk, Mathematical and numerical model for non-linear viscoplastictity, Phil. Trans. R. Soc. A, 369, 2864-2880, 2011. 
[5] E. Godlewski \& P.-A. Raviart, Hyperbolic systems of conservation laws Mathematics and Applications (Paris). Ellipses, vol. 3/4, 1991.

[6] P.D. Lax, Hyperbolic Partial Differential Equations Lecture notes, Courant Institute of Mathematical Sciences, American Mathematical Society, 2006.

[7] P.G. LeFloch, Hyperbolic Systems of Conservation Laws, The Theory of Classical and Nonclassical Shock Waves, Lectures in Mathematics, Birkhä user, 2002

[8] D. Serre, Systèmes de lois de conservation I. Fondations. Diderot Editeur, Paris, 1996.

[9] R. J. Flory, Thermodynamic relations for highly elastic materials Transactions of Faraday Society ,57, 829-838, 1981

[10] S.

Ndanou, N. Favrie \& S. Gavrilyuk, Criterion of hyperbolicity in hyperelasticity in the case of the stored energy in separable form, J. Elasticity 2013 http ://link.springer.com/journal/10659/onlineFirst/page/1

[11] A. Kulikovskii \& E. Sveshnikova, Nonlinear waves in elastic media, CRC Press, Boca Raton, FL, 1995. 